\title{
Approximate Analytical Solution for the Forced Korteweg-de Vries Equation
}

\author{
Vincent Daniel David, ${ }^{1,2}$ Mojtaba Nazari, ${ }^{1}$ Vahid Barati, ${ }^{1}$ \\ Faisal Salah, ${ }^{3,4}$ and Zainal Abdul Aziz ${ }^{1,3}$ \\ ${ }^{1}$ Department of Mathematical Sciences, Faculty of Science, Universiti Teknologi Malaysia, 81310 Johor Bahru, Johor, Malaysia \\ ${ }^{2}$ Faculty of Computer and Mathematical Sciences, Universiti Teknologi MARA, 40450 Shah Alam, Selangor, Malaysia \\ ${ }^{3}$ UTM Centre for Industrial and Applied Mathematics, Universiti Teknologi Malaysia, 81310 Johor Bahru, Johor, Malaysia \\ ${ }^{4}$ Department of Mathematics, Faculty of Science, University of Kordofan, North Kordofan State, El-Obied 51111, Sudan
}

Correspondence should be addressed to Zainal Abdul Aziz; abdulazizzainal@gmail.com

Received 17 June 2013; Accepted 10 November 2013

Academic Editor: Huijun Gao

Copyright (c) 2013 Vincent Daniel David et al. This is an open access article distributed under the Creative Commons Attribution License, which permits unrestricted use, distribution, and reproduction in any medium, provided the original work is properly cited.

The forced Korteweg-de Vries (fKdV) equations are solved using Homotopy Analysis Method (HAM). HAM is an approximate analytical technique which provides a novel way to obtain series solutions of such nonlinear problems. It has the auxiliary parameter $\hbar$, where it is easy to adjust and control the convergence region of the series solution. Some examples of forcing terms are employed to analyse the behaviours of the HAM solutions for the different $\mathrm{fKdV}$ equations. Finally, this form of HAM solution is compared with the analytical soliton-type solution of $\mathrm{fKdV}$ equation as derived by Zhao and Guo. The results is found to be in good agreement with Zhao and Guo.

\section{Introduction}

An analytical model of Tsunami propagation was proposed by Pelinovsky et al. [1] as follows:

$$
\frac{\partial \eta}{\partial t}+c \frac{\partial \eta}{\partial x}+\alpha \eta \frac{\partial \eta}{\partial x}+\beta \frac{\partial^{3} u}{\partial x^{3}}=\frac{\partial f}{\partial x}
$$

with

$$
\alpha=\frac{3 c}{2 h_{0}}, \quad \beta=\frac{c h_{0}^{2}}{6}, \quad f=\frac{-c z}{2},
$$

where $\eta=\eta(x, t)$ refers to the elevation of free water surface, $z=z(x, t)$ represents the solid bottom, $h$ is assumed to be the constant mean water depth, and $c \approx \sqrt{g h}$ is the long wave speed with $g$ being gravity acceleration. If the right-hand side of (1), that is, $\partial f / \partial x=0$, then (1) will be the Kortewegde Vries equation which is completely integrable [2]. But if $\partial f / \partial x \neq 0$, then the equation is difficult to be integrable where the term $\partial f / \partial x$ is called forcing term. Therefore, (1) is known as "KdV equation with forcing term" or "forced KdV (fKdV)" equation [3]. follows:

The $\mathrm{fKdV}$ equation to be considered in this paper is as

$$
\frac{\partial u}{\partial t}+\alpha u \frac{\partial u}{\partial x}+\beta \frac{\partial^{3} u}{\partial x^{3}}=\frac{\partial f}{\partial x},
$$

where $\alpha$ and $\beta$ are constant.

This form (3) is an alternative approach in describing the governing equations for the basic hydrodynamic model of Tsunami generation, for example, by atmospheric disturbances (Akylas [4] and Nosov and Skachko [5]). The appropriate evolution equation, which asymptotically approximates the Boussinesq equation, leads to this $\mathrm{fKdV}$ equation (3). The forcing term in the $\mathrm{fKdV}$ can be assumed to be derivable from atmospheric disturbances. Various forms of this equation have been extensively studied (see Grimshaw et al. [6], Pelinovsky et al. [7]) and numerical results show that the solution contains the set of solitary waves. Evidently, the behaviour of Tsunami waves on the open ocean is considerably more complicated than the solitary wave model; nonetheless this $\mathrm{fKdV}$ equation has been used in Yaacob et al. [8] as a simple mathematical model that could describe 
the Tsunami waves modelling. In the present paper, the above-mentioned considerations motivate us to investigate the approximate analytical solutions of the $\mathrm{fKdV}$ equation by using HAM.

Up to now, to the best of our knowledge, the solutions of $\mathrm{fKdV}$ equation of (3) can only be obtained by numerical or perturbation techniques $[3,9,10]$. Recently, the analytical solution of $\mathrm{fKdV}$ equation with a certain form of forcing term has been solved by Zhao and Guo [3], using Hirota direct method. Thus the main reason of this work is to solve $\mathrm{fKdV}$ equation by using the homotopy analysis method (HAM) for various forcing terms, including in [3]. This approximate analytical HAM solution will be used to be compared with the analytical soliton-type solution of Zhao and Guo [3]. This analysis would in a way demonstrate the effectiveness and the potential of HAM to solve difficult nonlinear equations.

The homotopy analysis method (HAM) is an analytic method to solve nonlinear partial differential problems, which was first introduced by Liao in 1992 [11]. It is known that HAM has greater flexibility in the selection of a proper set of base functions for the solution and a much simpler way in the control of the convergence rate and region compared to perturbation approach [12-19]. Furthermore, this analytic technique does not have restriction of nonperturbation methods, such as Lyapunov's artificial small parameter method, the $\delta$-expansion method, and Adomian's decomposition method [20]. The analytic technique had been applied successfully in many nonlinear problems in engineering and science $[21,22]$, for example, the magnetohydrodynamic flows of non-Newtonian fluids over a stretching sheet [23], nonlinear progressive waves [24], free oscillations of positively damped systems with algebraically decaying amplitude [25], free oscillations of self-excited systems [26], and similarity boundary layer equations [27].

The paper is organized as follows. The HAM of the $\mathrm{fKdV}$ equation (3) is presented in Section 2. Section 3 briefly illustrates the HAM solution of $\mathrm{KdV}$ equation. Section 4 is divided into 2 parts. The first (Section 4.1) discusses the concept of HAM and is followed by some examples of external forces employed on the $\mathrm{KdV}$ equation. Meanwhile, Section 4.2 compares the results of the analytical solution of [3] with the generated HAM solution. Conclusion is concisely laid out in Section 5.

\section{Basic Ideas of HAM}

We consider differential equation in the form of $u(r, t)$,

$$
N[u(r, t)]=0,
$$

where $N$ is the nonlinear operator and $u(r, t)$ is the unknown function.

Using concept of HAM, we construct the so-called zeroth-order deformation equation

$$
(1-q) \ell\left[\varphi(r, t ; q)-u_{0}(r, t)\right]=q \hbar H(r, t) N[\varphi(r, t ; q)],
$$

where $u_{0}(r, t)$, the initial guess of exact solution, $u(r, t) ; \hbar$, an auxiliary parameter with condition, $\hbar \neq 0 ; H$, an auxiliary function; $\ell$, an auxiliary linear operator; $q$, an embedding parameter with condition, $q \in[0,1]$.

Note that, when $q=0,(5)$ holds for $\varphi(r, t ; 0)=u_{0}(r, t)$ and when $q=1$, it holds for $\varphi(r, t ; 1)=u(r, t)$. As $q$ increases from 0 to 1 , the solution of $u(r, t, q)$ varies from the initial guess, $u_{0}(r, t)$, to the exact solution, $u(r, t)$.

By Taylor expansion of $\varphi(r, t ; q)$, in a power series with respect to $q$, we obtain

$$
\varphi(r, t ; q)=\varphi(r, t ; 0)+\sum_{m=1}^{\infty} u(r, t) q^{m}
$$

where

$$
u_{m}(r, t)=\frac{1}{m !} \frac{\partial^{m} \varphi(r, t ; q)}{\partial q^{m}} \mid q=0
$$

With appropriate property of $u_{0}(r, t), \hbar, H$, and $\ell$, the series of (6) is convergence at $q=1$, and one has

$$
u(r, t)=u_{0}(r, t)+\sum_{m=1}^{\infty} u_{m}(r, t) .
$$

Let us define the following vectors:

$$
\vec{u}_{n}(r, t)=\left\{u_{0}(r, t), u_{1}(r, t), \ldots, u_{n-1}(r, t), u_{n}(r, t)\right\} .
$$

Differentiating the zeroth-order deformation equation (5) $m$-times with respect to the embedding parameter $q$ and dividing it by $m$ ! and finally letting $q=0$, we obtain the following $m$ th-order deformation equation:

$$
\ell\left[u_{m}(r, t)-\chi_{m} u_{m-1}(r, t)\right]=\hbar H(r, t) R_{m}\left[u_{m-1}^{\vec{m}}(r, t)\right],
$$

where

$$
\begin{gathered}
\chi_{m}= \begin{cases}1, & \text { when } m>1, \\
0, & \text { otherwise, }\end{cases} \\
R_{m}\left[u_{m-1}^{\rightarrow}(r, t)\right] \\
=\left.\frac{1}{(m-1) !}\left\{\frac{\partial^{m-1}}{\partial q^{m-1}} N\left[\sum_{m=0}^{\infty} u_{m}(r, t) q^{m}\right]\right\}\right|_{q=0} .
\end{gathered}
$$

It is noted that HAM contains control-convergence parameter $\hbar$ where it provides an easy way to adjust and control the convergence region. This can be done by plotting the $\hbar$ curves.

\section{HAM Solution on KdV}

When the forcing term of (3) becomes zero, then the equation becomes the Korteweg-de Vries (KdV) equation. Wazwaz [2] gave a form of the exact solution of KdV equation. Nazari et al. [28] used HAM to obtain a comparable approximate analytical solution of the $\mathrm{KdV}$ equation, which is in good 


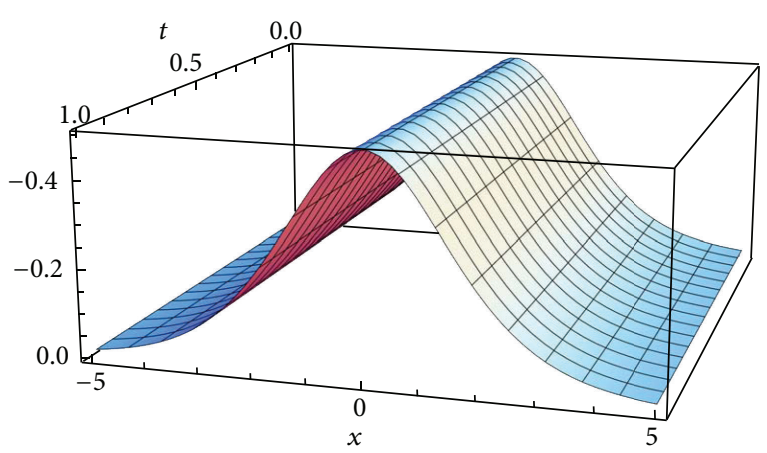

FIGURE 1: The HAM solution of KdV whereby forcing term is zero.

agreement with Wazwaz's exact solution of KdV equation. Figure 1 is given the graphical representation of the HAM solution of $\mathrm{KdV}$ equation, where the forcing term of (3) is zero; $\partial f / \partial x=0$.

Further to this research output, we will now apply HAM to solve different $\mathrm{fKdV}$ equations based on various forcing terms.

\section{Approximate Analytical Solution for fKdV Using HAM}

For HAM, the zeroth-order deformation equation is given by

$$
(1-q) \ell\left[u(x, t ; q)-u_{0}(x, t)\right]=q \hbar H(x, t) N[u(x, t ; q)]
$$

and we use

$$
u_{0}(x, t)=\frac{-2 e^{x}}{\left(1+e^{x}\right)^{2}}
$$

as the initial guess and

$$
\ell[\varphi(x, t ; q)]=\frac{\partial u(x, t ; q)}{\partial t}
$$

as the auxiliary linear operator, which satisfies

$$
\ell[c]=0,
$$

where $c$ is constant.

Considering

$$
\begin{gathered}
H(x, t)=1, \\
N[u(x, t ; q)]=\frac{\partial u(x, t ; q)}{\partial t}+\alpha u(x, t ; q) \frac{\partial u(x, t ; q)}{\partial x} \\
+\beta \frac{\partial^{3} u(x, t ; q)}{\partial x^{3}}-\frac{\partial f}{\partial x},
\end{gathered}
$$

the $m$ th-order deformation problem now becomes

$$
\begin{aligned}
& \ell\left[u_{m}(x, t)-\chi_{m} u_{m-1}(x, t)\right] \\
& \quad=q \hbar\left[\frac{\partial u_{m-1}}{\partial t}+\alpha\left(\sum_{i=0}^{m-1} u_{i} \frac{\partial u_{m-1-i}}{\partial x}\right)+\beta \frac{\partial^{3} u_{m-1}}{\partial x^{3}}-\frac{\partial f_{m-1}}{\partial x}\right]
\end{aligned}
$$

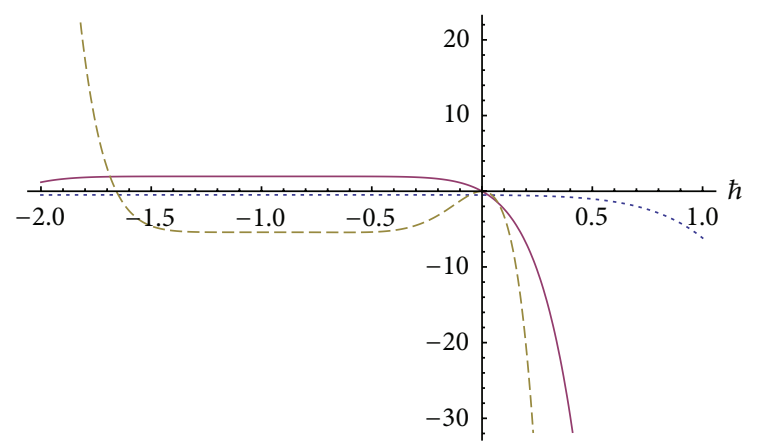

FIgURE 2: The $\hbar$-curves according to 10th-order approximation. Dashed point: $u(0.01,0.01)$, solid line: $\dot{u}(0.01,0.01)$, and dashed line: $\ddot{u}(0.01,0.01)$.

with

$$
u_{m}(x, 0)=0 \text { for } m>1
$$

4.1. Using Different Forcing Terms for $f K d V$. For simplicity, we let $\alpha=-6$ and $\beta=1$ for all the cases studied below.

4.1.1. Forcing Term, $\partial f / \partial x=x^{2}$. Consider the nonlinear KdV equation with the quadratic term $x^{2}$ as the forcing term.

Then (3) is simplified as follows:

$$
\frac{\partial u}{\partial t}-6 u \frac{\partial u}{\partial x}+\frac{\partial^{3} u}{\partial x^{3}}=x^{2} .
$$

In this paper, MATHEMATICA ${ }^{\odot}$ is used to solve the nonlinear differential equation (16) for the case $\partial f / \partial x=x^{2}$.

It is found that the solution for 10th-order approximation is

$$
\begin{aligned}
u(x, t)= & -\frac{2 e^{x}}{\left(1+e^{x}\right)^{2}} \\
& -\frac{\hbar t\left(2 e^{x}-2 e^{2 x}+x^{2}+3 e^{x} x^{2}+3 e^{2 x} x^{2}+e^{3 x} x^{2}\right)}{\left(1+e^{x}\right)^{3}} \\
& +\cdots .
\end{aligned}
$$

Liao [11] pointed out that the valid region of $\hbar$ is a horizontal line segment. Looking at $\hbar$-curves in Figure 2 , the valid region of convergence interval is $-1.5<\hbar<-0.4$. We choose three different values of $\hbar$ in the valid interval. The outcomes for the first case are shown in Figure 3.

The HAM solution at 10th-order approximation is presented with various values of $\hbar$, where convergence interval is shown in Figure 3. Figures 3(a) and 3(b) obtained for $\hbar=$ -0.6 , where the interval for Figure 3(a) is $0 \leq t \leq 0.2$ and for Figure 3(b) is $0 \leq t \leq 0.3$, respectively. It is observed that there is a slight formation on the waves when time had been increased from $t$ is $0.2 \mathrm{~s}$ to $0.3 \mathrm{~s}$. The phenomenon is similar to the result for $\hbar=-1$ of Figures $3(\mathrm{c})$ and $3(\mathrm{~d})$. 


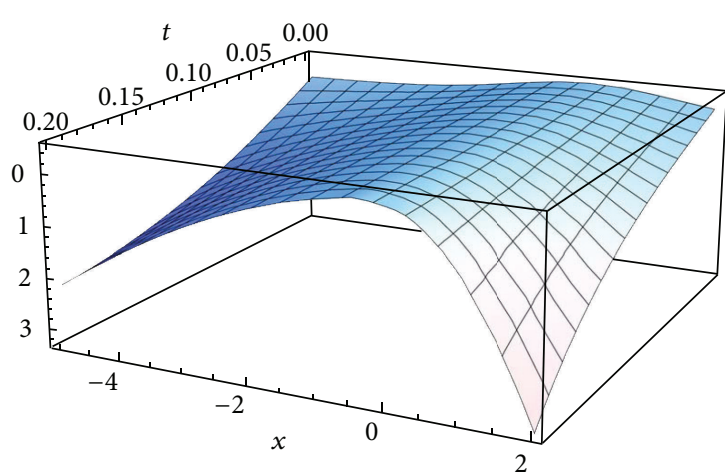

(a)

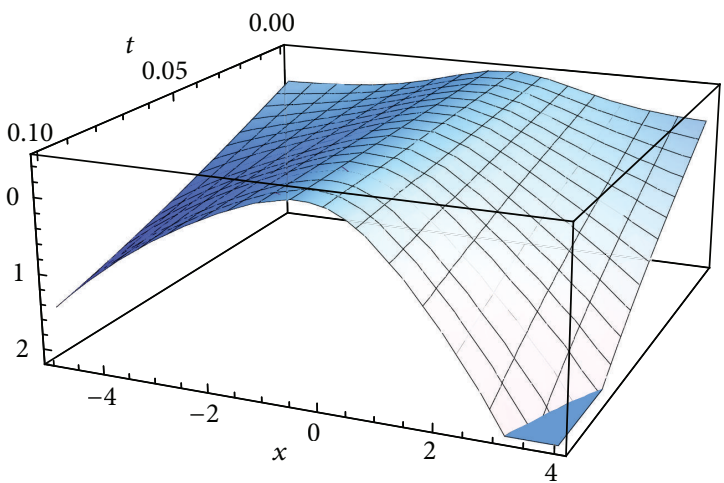

(c)

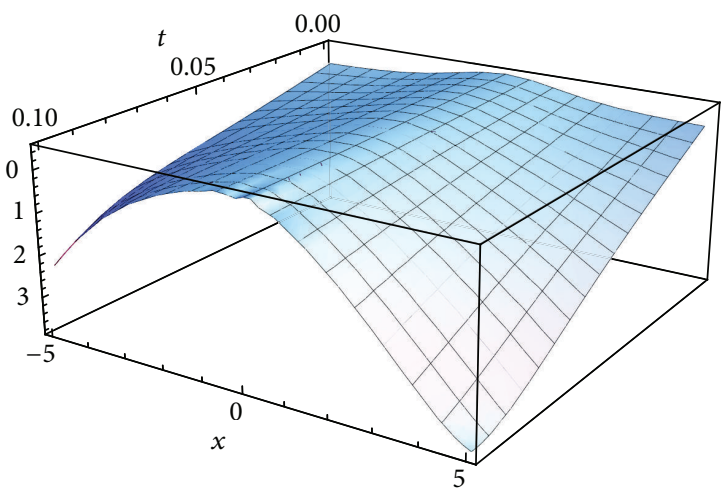

(e)

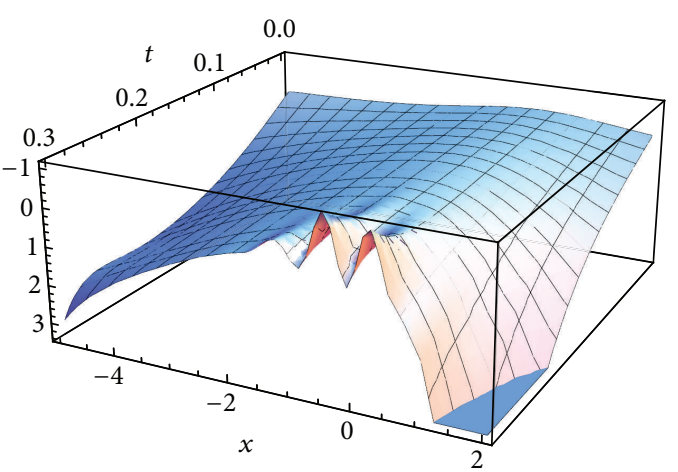

(b)

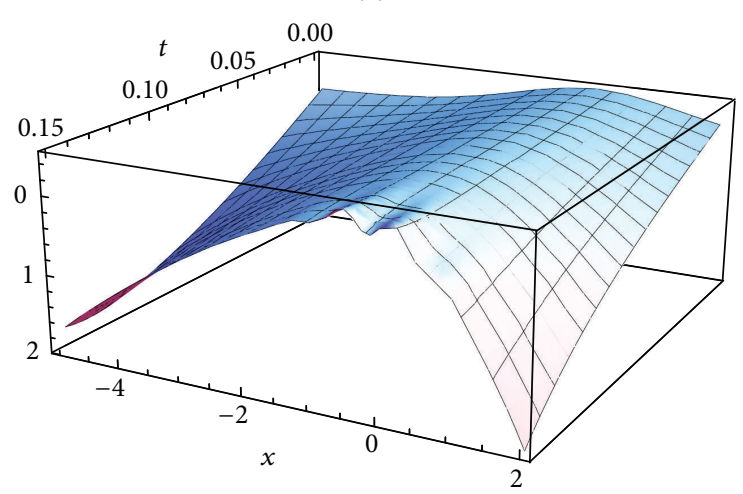

(d)

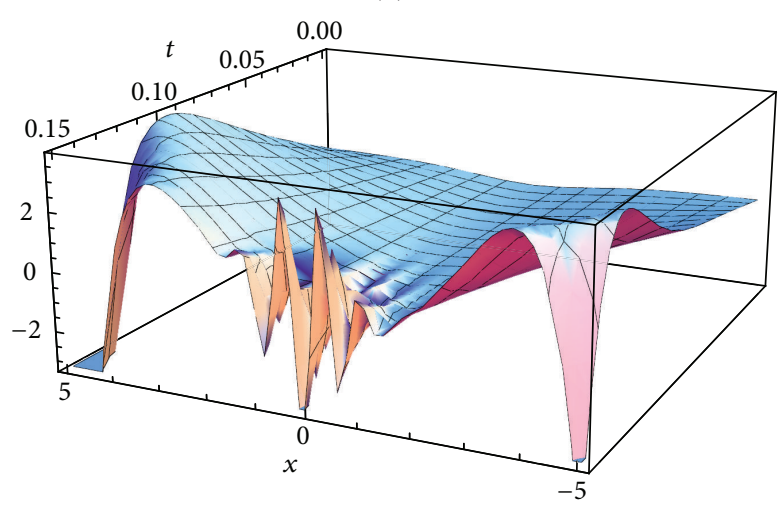

(f)

FIGURE 3: The HAM solution at 10th-order approximation with different convergence values of $\hbar$. (a and b) for $\hbar=-0.6,(\mathrm{c}$ and $\mathrm{d}$ ) for $\hbar=-1.0$, and (e and $\mathrm{f}$ ) for $\hbar=-1.4$.

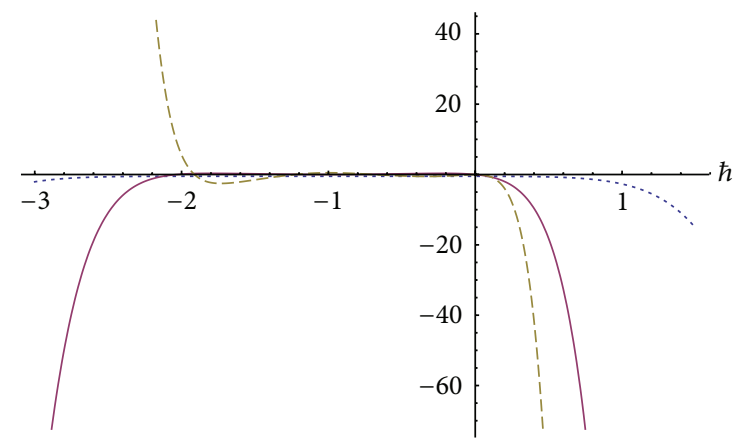

FIGURE 4: The $\hbar$-curves according to 9th-order approximation. Dashed point: $u(0.01,0.01)$, solid line: $\dot{u}(0.01,0.01)$, and dashed line: $\ddot{u}(0.01,0.01)$.
It is noted that the wave is normal with no turbulence in Figure $3(\mathrm{e})$ where then $\hbar$ value is chosen to be 1.4. Comparing Figures $3(\mathrm{e})$ and $3(\mathrm{f})$ with the same value of $\hbar$, it is observed that the wave exhibits anomalous behaviour when time is increased. For all cases above, we found that the wave displays certain chaotic manner when we increase the time. Figure 3 shows clearly that the wave changes in abnormal mode corresponding to the quadratic force in the right-hand side of (18).

4.1.2. Forcing Term, $\partial f / \partial x=\sin (x)$. Consider the nonlinear $\mathrm{KdV}$ equation with the quadratic term $\sin (x)$ as the forcing term. 


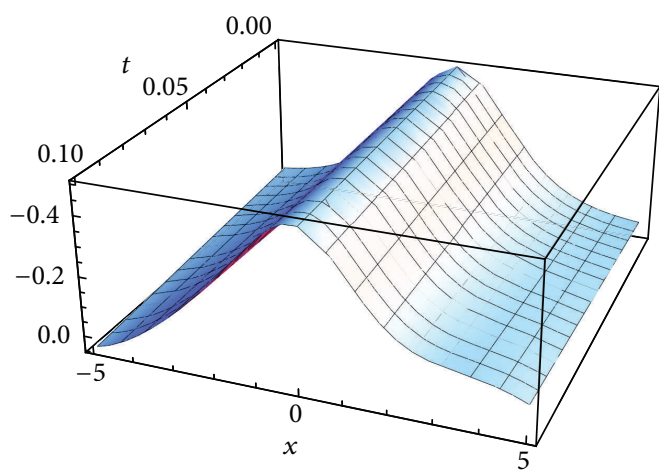

(a)

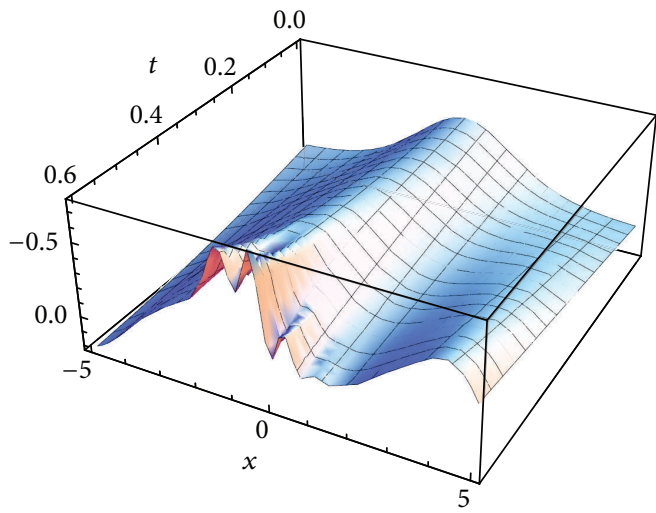

(c)

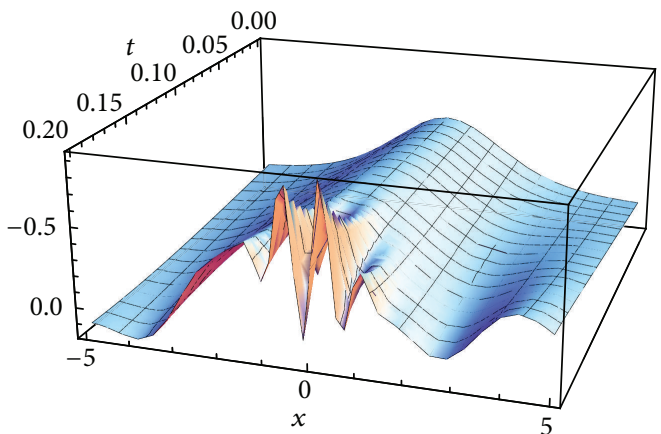

(e)

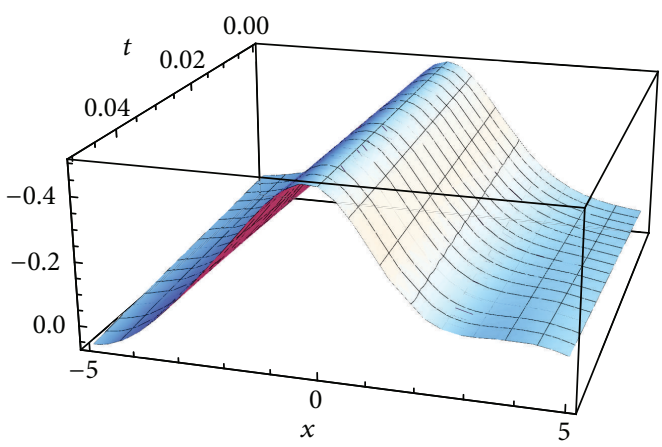

(g)

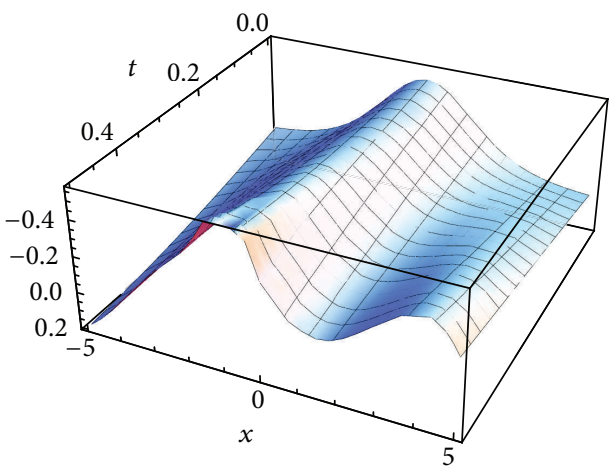

(b)

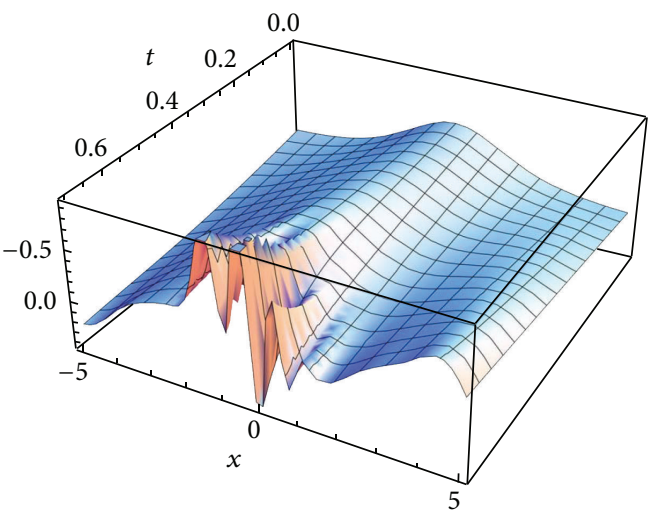

(d)

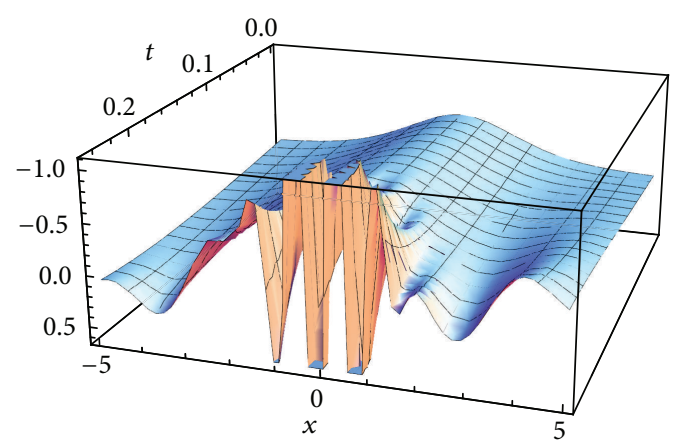

(f)

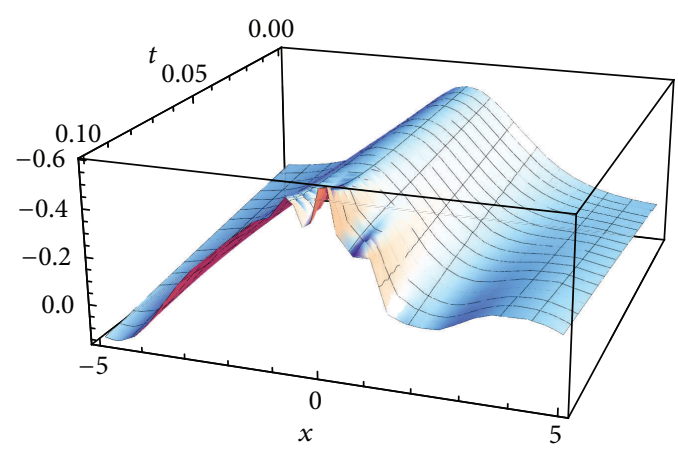

(h)

FIGURE 5: The HAM solution at 9th-order approximation with different convergence values of $\hbar$. (a, b, c, and d) for $\hbar=-0.3$, (e and f) for $\hbar=-1.0$, and $(\mathrm{g}$ and $\mathrm{h})$ for $\hbar=-1.7$. 


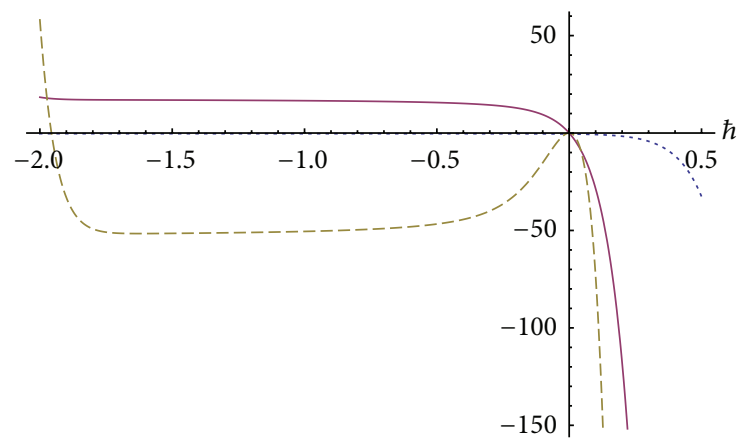

Figure 6: The $\hbar$-curves according to 17 th-order approximation. Dashed point: $u(0.01,0.01)$, solid line: $\dot{u}(0.01,0.01)$, and dashed line: $\ddot{u}(0.01,0.01)$.

The $\mathrm{fKdV}$ equation is as follows:

$$
\frac{\partial u}{\partial t}-6 u \frac{\partial u}{\partial x}+\frac{\partial^{3} u}{\partial x^{3}}=\sin (x) .
$$

Similarly MATHEMATICA ${ }^{\odot}$ is used to solve the nonlinear differential equation (16) for the case $\partial f / \partial x=\sin (x)$.

The solution at 9 th-order approximation is

$$
\begin{aligned}
u(x, t)= & -\frac{2 e^{x}}{\left(1+e^{x}\right)^{2}} \\
& -\hbar t\left(2 e^{x}-2 e^{2 x}+\sin (x)+3 e^{x} \sin (x)\right. \\
& \left.+3 e^{2 x} \sin (x)+e^{3 x} \sin (x)\right) \\
& \times\left(\left(1+e^{x}\right)^{3}\right)^{-1}+\cdots .
\end{aligned}
$$

Liao [11] pointed out that the horizontal line segment will be the valid region of $\hbar$. Based on Figure 4 , the admissible interval will be $-1.7<\hbar<-0.3$, so we choose $\hbar=-0.3$, $\hbar=-1$, and $\hbar=-1.7$.

The outcomes for the $\sin (x)$ force term are shown in Figure 5.

Figure 5 shows the HAM solution at 9th-order approximation with three different convergent values of $\hbar$. Figures 5(a), 5(b), 5(c), and 5(d) are the outcomes for different intervals of time less than $\hbar=-0.3$. The interval for Figure 5(a) is $0 \leq t \leq 0.1$ and the wave portrays a normal wave without any disturbance. The wave seems to be disturbed and the transition seen clearly in Figures 5(b) and 5(c) after the time is increased. In Figure 5(d), the time is increased to $0.7 \mathrm{~s}$ and it is observed that the wave starts to fluctuate. Upon considering $\hbar=-1$, the output is shown in Figures 5(e) and 5(f) where the suddenness and large energy of waves are created in short period of time. Figures $5(\mathrm{~g})$ and $5(\mathrm{~h})$ are the outcomes of $\hbar=-1.7$ for $0 \leq t \leq 0.06$ and $0 \leq t \leq$ 0.1 where the waves are compressed and propagate in short time. As a whole it can be concluded that the wave changes tremendously over time with the sine force incorporated in $\mathrm{fKdV}$ equation.
4.1.3. Forcing Term, $\partial f / \partial x=e^{x}$. Consider the nonlinear $\mathrm{KdV}$ equation with the quadratic term $e^{x}$ as the forcing term.

The $\mathrm{fKdV}$ equation is as follows:

$$
\frac{\partial u}{\partial t}-6 u \frac{\partial u}{\partial x}+\frac{\partial^{3} u}{\partial x^{3}}=e^{x}
$$

Similarly MATHEMATICA $\left.{ }^{(}\right)$is used to solve the nonlinear differential equation (16) for the case $\partial f / \partial x=e^{x}$.

The solution at 17 th-order approximation is

$$
\begin{aligned}
& u(x, t)=-\frac{2 e^{x}}{\left(1+e^{x}\right)^{2}}- \frac{e^{x}\left(3+e^{x}+3 e^{2 x}+e^{3 x}\right) \hbar t}{\left(1+e^{x}\right)^{3}} \\
&-\frac{e^{x} \hbar t}{2\left(1+e^{x}\right)^{4}}\left(8+16 e^{x}+20 e^{2 x}+16 e^{3 x}\right. \\
&+4 e^{4 x}+6 \hbar+8 e^{x} \hbar+8 e^{2 x} \hbar \\
&+8 e^{3 x} \hbar+2 e^{4 x} \hbar+3 \hbar t+20 e^{x} \hbar t \\
&\left.+32 e^{2 x} \hbar t+4 e^{3 x} \hbar t+e^{4 x} \hbar t\right)+\cdots
\end{aligned}
$$

Based on Figure 6, the accepted convergence interval will be $-1.7 \leq \hbar \leq-0.5$. For further deliberation, the convergence values of $\hbar$ are chosen as, $\hbar=-0.5, \hbar=-1$, and $\hbar=-1.7$.

The outcomes for the $e^{x}$ force term are shown in Figure 7.

Figure 7 presents the solution of HAM at 17th-order approximationwith different convergence values of $\hbar$. Figures $7(\mathrm{a})$ and $7(\mathrm{~b})$ are observed for $\hbar=-0.5$ under the time interval of $0 \leq t \leq 0.001$ and $0 \leq t \leq 0.05$, respectively. The wave is undisturbed when $t$ is $0.001 \mathrm{~s}$ but the wave changes over time. This similar scenario occurs in Figure 7(c), upon using $\hbar=-1.0$. When $\hbar=-1.7$ is used the outcome is depicted in Figure 7(d) for the interval of $0 \leq t \leq 0.01$. It is found that the wave steadily rises over at a point in Figure $7(d)$.

4.2. Comparison of $f K d V$ HAM Solution with Zhao and Guo's Analytical Solution. In 2009, Zhao and Guo [3] gave a number of analytical solutions corresponding to the $\mathrm{fKdV}$ equation by using Hirota's direct method. In this paper, we will compare the analytical solution of [3] with the corresponding HAM solution. This comparison would again reveal the effectiveness and potential of HAM to solve difficult nonlinear equations.

The $\mathrm{fKdV}$ equation used in [3] is as follows:

$$
\frac{\partial u}{\partial t}+u \frac{\partial u}{\partial x}+\lambda \frac{\partial^{3} u}{\partial x^{3}}=\varphi \frac{\partial f}{\partial x}
$$

with

$$
\begin{array}{r}
f=\frac{12 k \lambda}{\varphi}\left(k^{3}(4 \lambda-\phi)-\frac{\partial a(t)}{\partial t}\right) \\
\times \operatorname{sech}^{2}\left(k\left(x-\phi k^{2} t\right)-a(t)\right), \\
a(t)=b \arctan (A t)+b_{1} t+b_{0} .
\end{array}
$$




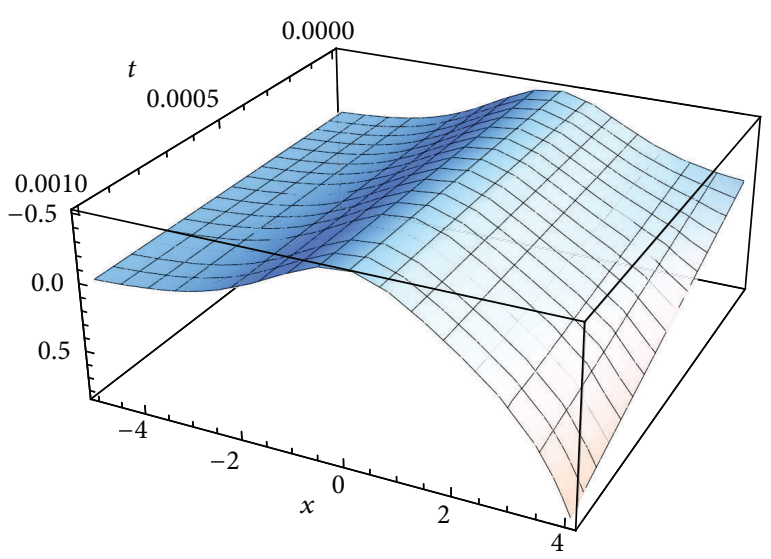

(a)

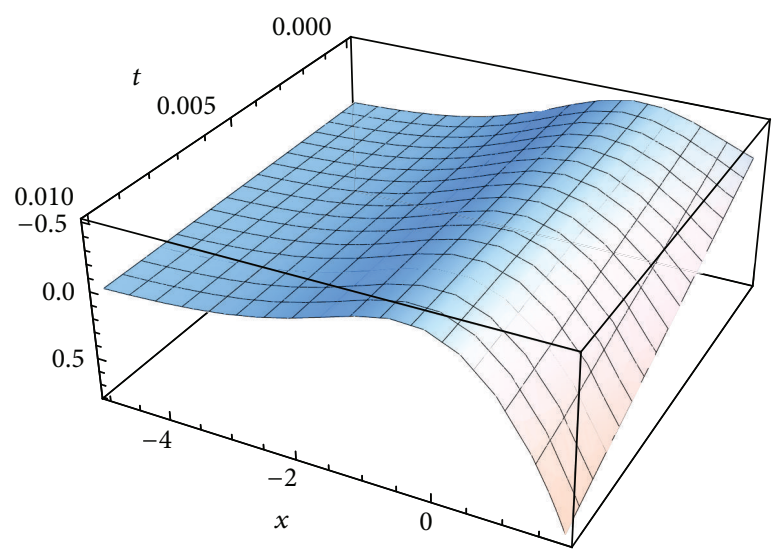

(c)

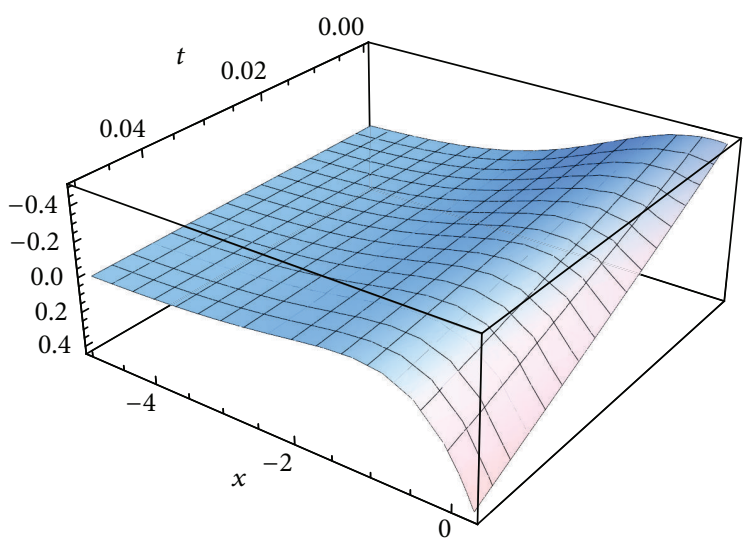

(b)

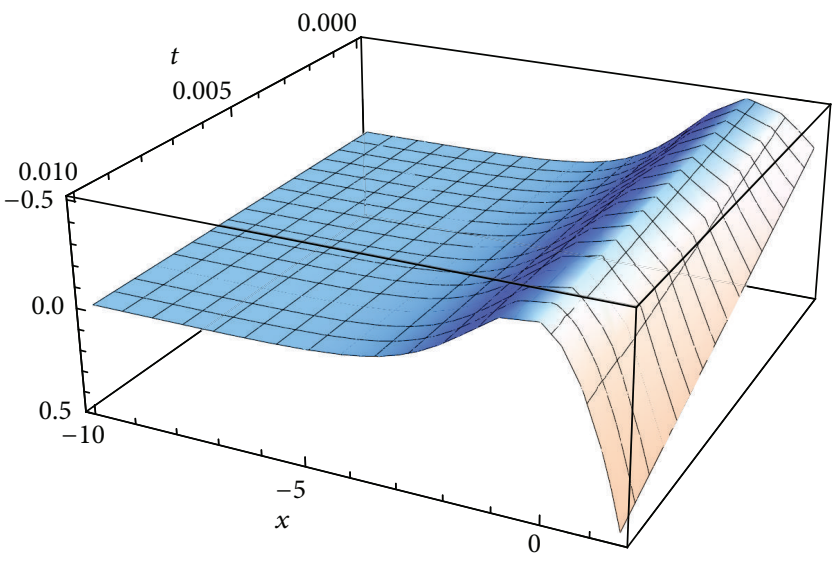

(d)

FIGURE 7: The HAM solution at 17th-order approximation with different convergence values of $\hbar$. (a and b) for $\hbar=-0.5$, (c) for $\hbar=-1.0$, and (d) for $\hbar=-1.7$.

The parameters chosen by Zhao and Guo [3] and the same input are used in our HAM procedure and are shown in Figure 8, that are

$$
\begin{aligned}
& k=1, \quad \phi=4, \quad \lambda=\frac{1}{4}, \quad \varphi=2, \\
& A=3, \quad b=-1, \quad b_{1}=-3, \quad b_{0}=0 .
\end{aligned}
$$

Figure 8 shows the comparison between the graphs of analytical solution of [3] with the HAM solution. Due to very high computational and some limitations on technologies being used, we have simplified (25), by making the term $t$ as constant in the forcing term. For purpose of comparison with the result of [3], we have chosen to show four cases at $t=$ $0.25 \mathrm{~s}, 0.5 \mathrm{~s}, 0.75 \mathrm{~s}$, and $1 \mathrm{~s}$. It should be noted that, since the time has been fixed as constant in the right-hand side term, then for each case of different $\hbar$, the ensuing different orders of approximation are obtained. HAM solution shows perfect agreement at $u(x, 1)$ with the analytical solution of [3]. As a whole, based on the graphs obtained, HAM solution shows good approximation with the analytical solution of [3].

\section{Conclusion}

In this paper, HAM is used to get the approximate analytical solutions of forced $\mathrm{KdV}$ equations for different applied forcing terms. With the existence of auxiliary parameter $\hbar$, HAM provides us with a straightforward way to control the convergence of approximation series whereby it makes a noticeable difference between HAM and the existing perturbation methods. Figures 2-7 show distinct fluctuations in the wave profiles of the HAM solutions, with various forcing terms being incorporated in the $\mathrm{fKdV}$ equations. Nonetheless, Figure 8 shows very close approximation of HAM solution with the analytical solution of Zhao and Guo [3]. These outcomes bring to light that HAM has flexibility and potential to solve complicated nonlinear problems. Since $\mathrm{fKdV}$ equation is associated with modelling of Tsunami propagation, as proposed by Pelinovsky et al. [1], we are hopeful that this work would have some added insight into more complicated Tsunami propagation models. In order to achieve more practical oriented results, there are suggestions that further work could be considered under data-driven (measurements) framework, for example, a recent overview can be seen from [29]. 


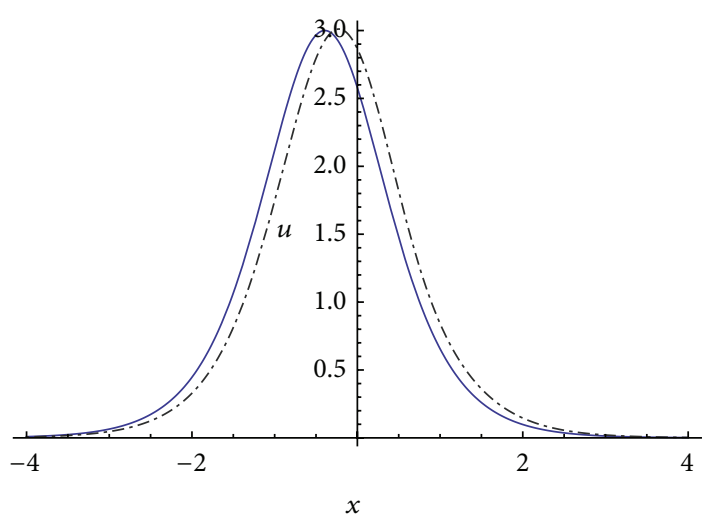

$u(x, 0.25)$ at 10th-order approximation with $\hbar=-1.6$

(a)

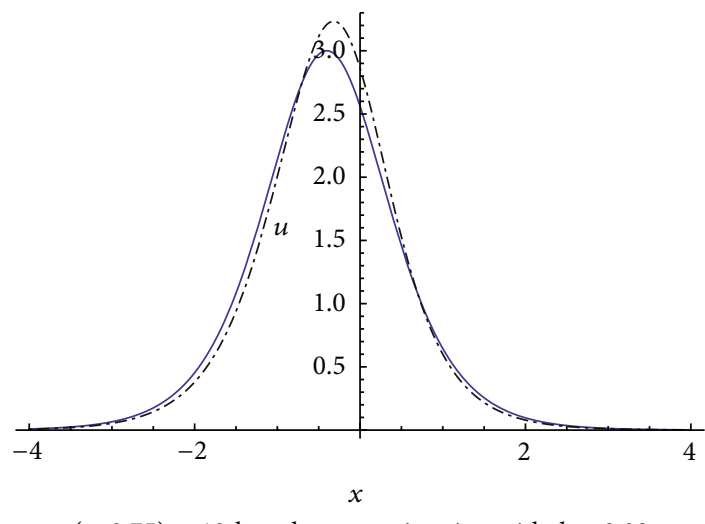

$u(x, 0.75)$ at 10 th-order approximation with $\hbar=0.08$

(c)

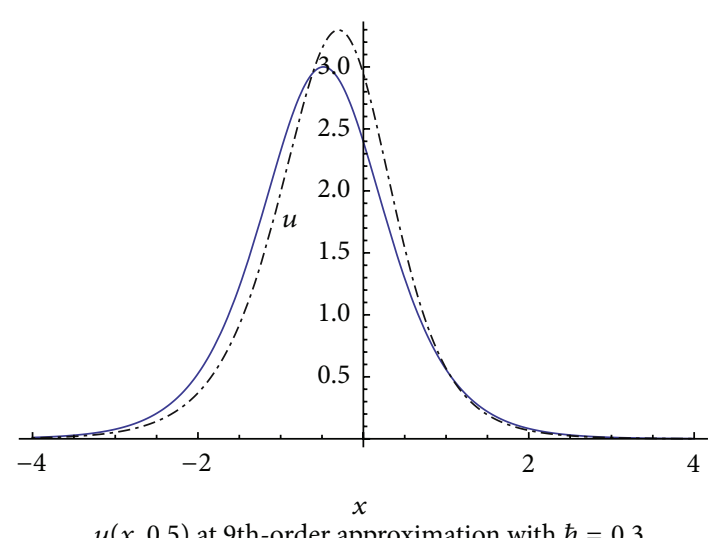

$u(x, 0.5)$ at 9 th-order approximation with $\hbar=0.3$

(b)

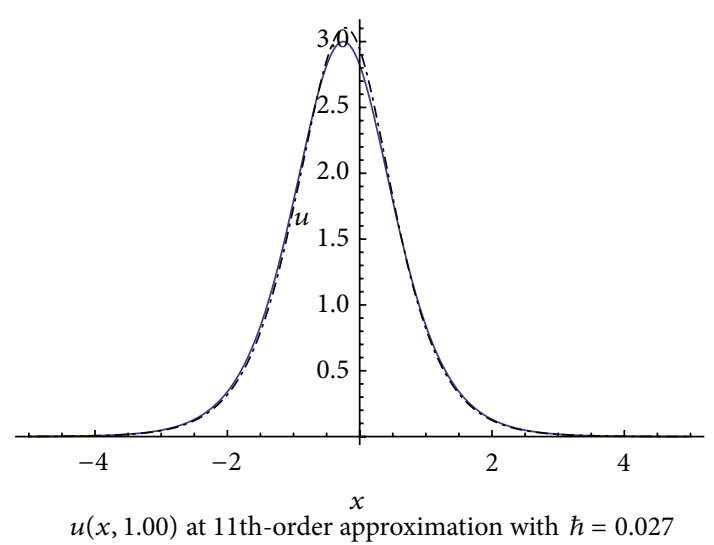

(d)

FIGURE 8: MATHEMATICA ${ }^{\odot}$ graphs depicting $u(x, 0.25), u(x, 0.50), u(x, 0.75)$, and $u(x, 1)$ against $x$. Solid line is for analytical solution of [3], and dash line is the HAM solution.

\section{Acknowledgments}

V. D. David is thankful for the Ministry of Higher Education (MOHE) and Universiti Teknologi MARA Malaysia (UiTM Malaysia) for the research scholarship. M. Nazari is thankful for UTM for the International Doctoral Fellowship (IDF). F. Salah is thankful for UTM for the postdoctoral fellowship and University of Kordofan for the research leave. This research is partially funded by UTM-CIAM Flagship Project Grant, Vote no. PY/2013/00474.

\section{References}

[1] E. N. Pelinovsky, A. C. Yalciner, E. Okal, and C. E. Synolakis, Eds., Submarine Landslides and Tsunamis, vol. 21, Springer, Berlin, Germany, 2003.

[2] A. M. Wazwaz, "Construction of solitary wave solutions and rational solutions for the KdV equation by Adomian decomposition method," Chaos, Solitons and Fractals, vol. 12, no. 12, pp. 2283-2293, 2001.

[3] J.-X. Zhao and B.-L. Guo, "Analytic solutions to forced KdV equation," Communications in Theoretical Physics, vol. 52, no. 2, pp. 279-283, 2009.
[4] T. R. Akylas, "On the excitation of long nonlinear water waves by a moving pressure distribution," Journal of Fluid Mechanics, vol. 141, pp. 455-466, 1984.

[5] M. A. Nosov and S. N. Skachko, "Nonlinear mechanism of tsunami generation by bottom oscillations," Natural Hazard and Earth Sciences, no. 1, pp. 44-47, 2001.

[6] R. Grimshaw, E. Pelinovsky, and X. Tian, "Interaction of a solitary wave with an external force," Physica $D$, vol. 77 , no. 4 , pp. 405-433, 1994.

[7] E. Pelinovsky, T. Talipova, A. Kurkin, and C. Kharif, "Nonlinear mechanism of tsunami wave generation by atmospheric disturbances," Natural Hazard and Earth Sciences, vol. 1, pp. 243-250, 2001.

[8] N. Yaacob, N. M. Sarif, and Z. A. Aziz, "Modelling of tsunami waves," MATEMATIKA, vol. 24, no. 2, pp. 211-230, 2008.

[9] E. Babolian, J. Saeidian, and A. Azizi, "Application of homotopy perturbation method to some nonlinear problems," Applied Mathematical Sciences, vol. 3, no. 45-48, pp. 2215-2226, 2009.

[10] H. Kim, H. J. Park, and D. Yoon, "Numerical stability analysis of steady solutions for the forced $\mathrm{KdV}$ equation based on the polynomial chaos expansion," European Journal of Mechanics $B$, vol. 39, pp. 71-86, 2012.

[11] J. S. Liao, Beyond perturbation, vol. 2, Chapman \& Hall, Boca Raton, Fla, USA, 2004. 
[12] S. J. Liao, The proposed homotopy analysis technique for the solution of nonlinear problems [Ph.D. thesis], Jiao Tong University, 1992.

[13] S. Liao and Y. Tan, "A general approach to obtain series solutions of nonlinear differential equations," Studies in Applied Mathematics, vol. 119, no. 4, pp. 297-354, 2007.

[14] S. Liao, "Comparison between the homotopy analysis method and homotopy perturbation method," Applied Mathematics and Computation, vol. 169, no. 2, pp. 1186-1194, 2005.

[15] S. Liao, "Notes on the homotopy analysis method: some definitions and theorems," Communications in Nonlinear Science and Numerical Simulation, vol. 14, no. 4, pp. 983-997, 2009.

[16] C. Wang, Y.-Y. Wu, and W. Wu, "Solving the nonlinear periodic wave problems with the homotopy analysis method," Wave Motion, vol. 41, no. 4, pp. 329-337, 2005.

[17] Y. Tan and S. Abbasbandy, "Homotopy analysis method for quadratic Riccati differential equation," Communications in Nonlinear Science and Numerical Simulation, vol. 13, no. 3, pp. 539-546, 2008.

[18] M. Nazari, F. Salah, and Z. A. Aziz, "Analytic approximate solution for the $\mathrm{KdV}$ equation with the homotopy analysis method," Matematika, vol. 28, no. 1, pp. 53-61, 2012.

[19] A. H. Salas, "Computing solutions to a forced KdV equation," Nonlinear Analysis: Real World Applications, vol. 12, no. 2, pp. 1314-1320, 2011.

[20] R. Ezzati and M. Aqhamohamadi, "Using homotopy analysis method to obtain approximate analytical solutions of wave equations," International Journal of Physical Sciences, vol. 6, no. 15, pp. 3821-3826, 2011.

[21] S. Abbasbandy, "The application of homotopy analysis method to nonlinear equations arising in heat transfer," Physics Letters A, vol. 360, no. 1, pp. 109-113, 2006.

[22] S. Abbasbandy, "The application of homotopy analysis method to solve a generalized Hirota-Satsuma coupled KdV equation," Physics Letters A, vol. 361, no. 6, pp. 478-483, 2007.

[23] S.-J. Liao, "On the analytic solution of magnetohydrodynamic flows of non-Newtonian fluids over a stretching sheet," Journal of Fluid Mechanics, vol. 488, pp. 189-212, 2003.

[24] S.-J. Liao and K. F. Cheung, "Homotopy analysis of nonlinear progressive waves in deep water," Journal of Engineering Mathematics, vol. 45, no. 2, pp. 105-116, 2003.

[25] S.-J. Liao, "An analytic approximate technique for free oscillations of positively damped systems with algebraically decaying amplitude," International Journal of Non-Linear Mechanics, vol. 38, no. 8, pp. 1173-1183, 2003.

[26] S.-J. Liao, "An analytic approximate approach for free oscillations of self-excited systems," International Journal of NonLinear Mechanics, vol. 39, no. 2, pp. 271-280, 2004.

[27] S.-J. Liao and I. Pop, "Explicit analytic solution for similarity boundary layer equations," International Journal of Heat and Mass Transfer, vol. 47, no. 1, pp. 75-85, 2004.

[28] M. Nazari, F. Salah, A. A. Zainal, and M. Nilashi, "Approximate analytic solution for the KdV and Burger equations with the homotopy analysis method," Journal of Applied Mathematics, vol. 2012, Article ID 878349, 13 pages, 2012.

[29] S. Yin, S. X. Ding, A. Haghani, H. Hao, and P. Zhang, "A comparison study of basic data-driven fault diagnosis and process monitoring methods on the benchmark Tennessee Eastman process," Journal of Process Control, vol. 22, no. 9, pp. 1567-1581, 2012. 


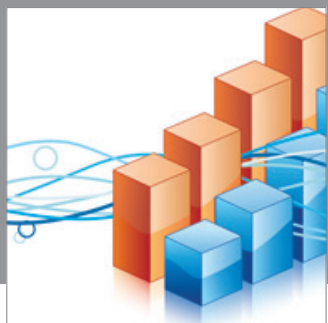

Advances in

Operations Research

mansans

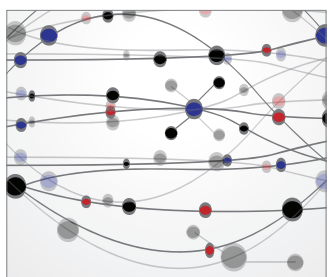

The Scientific World Journal
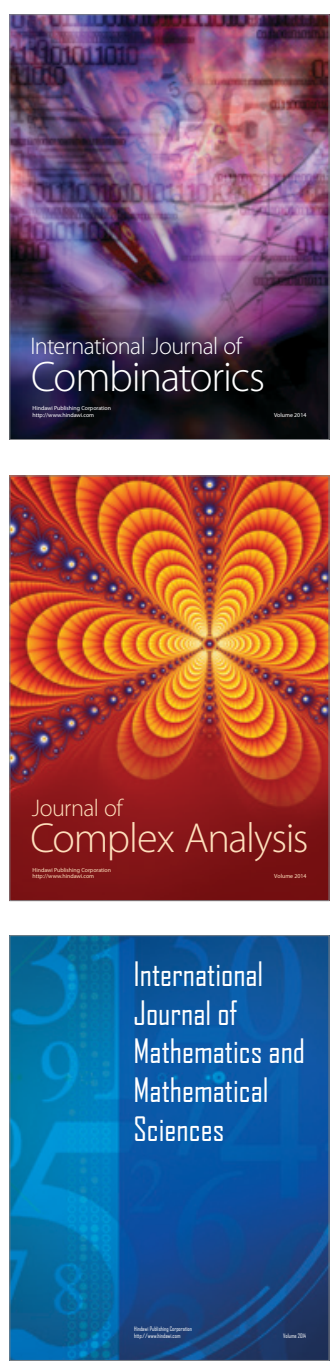
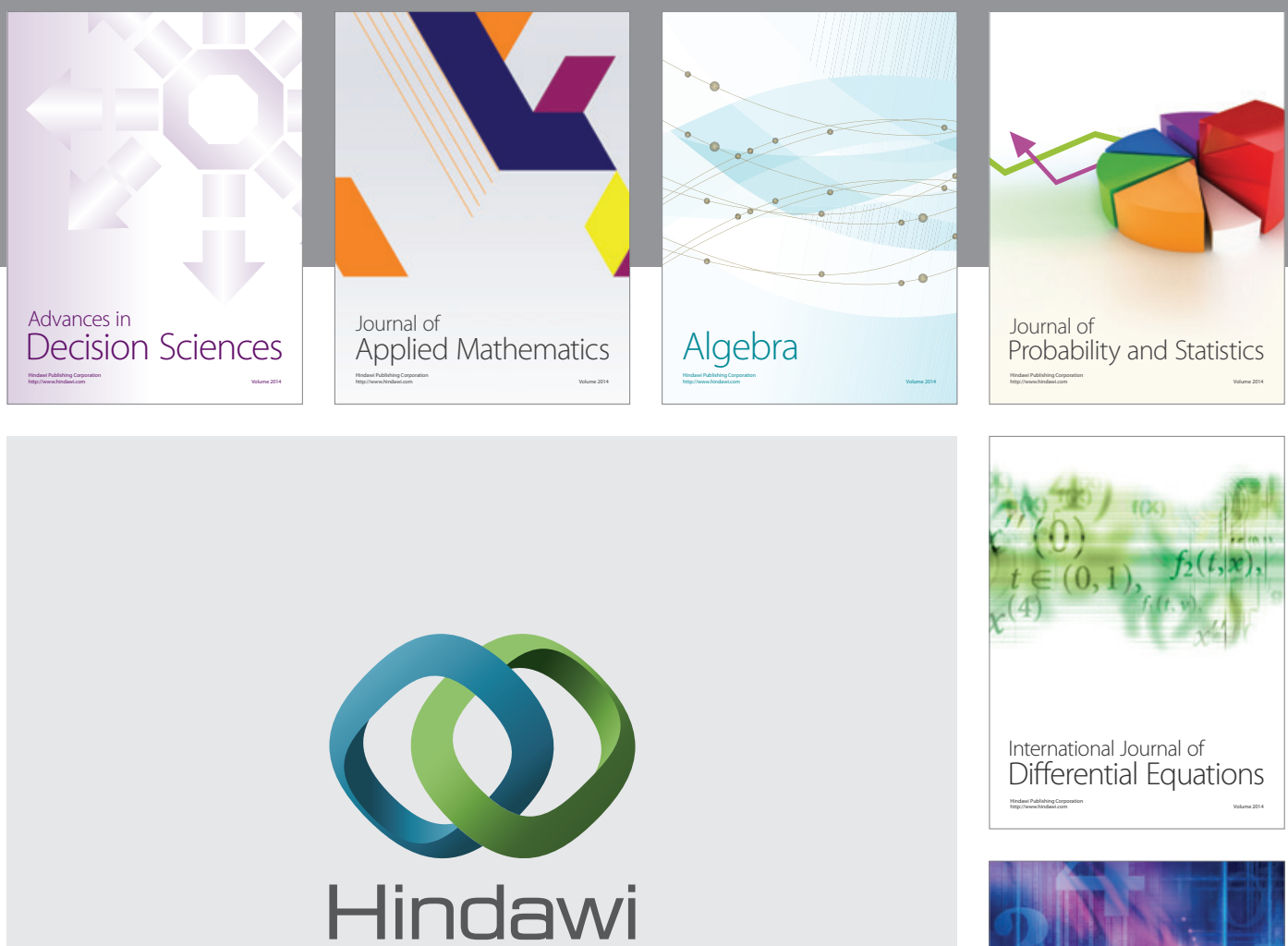

Submit your manuscripts at http://www.hindawi.com
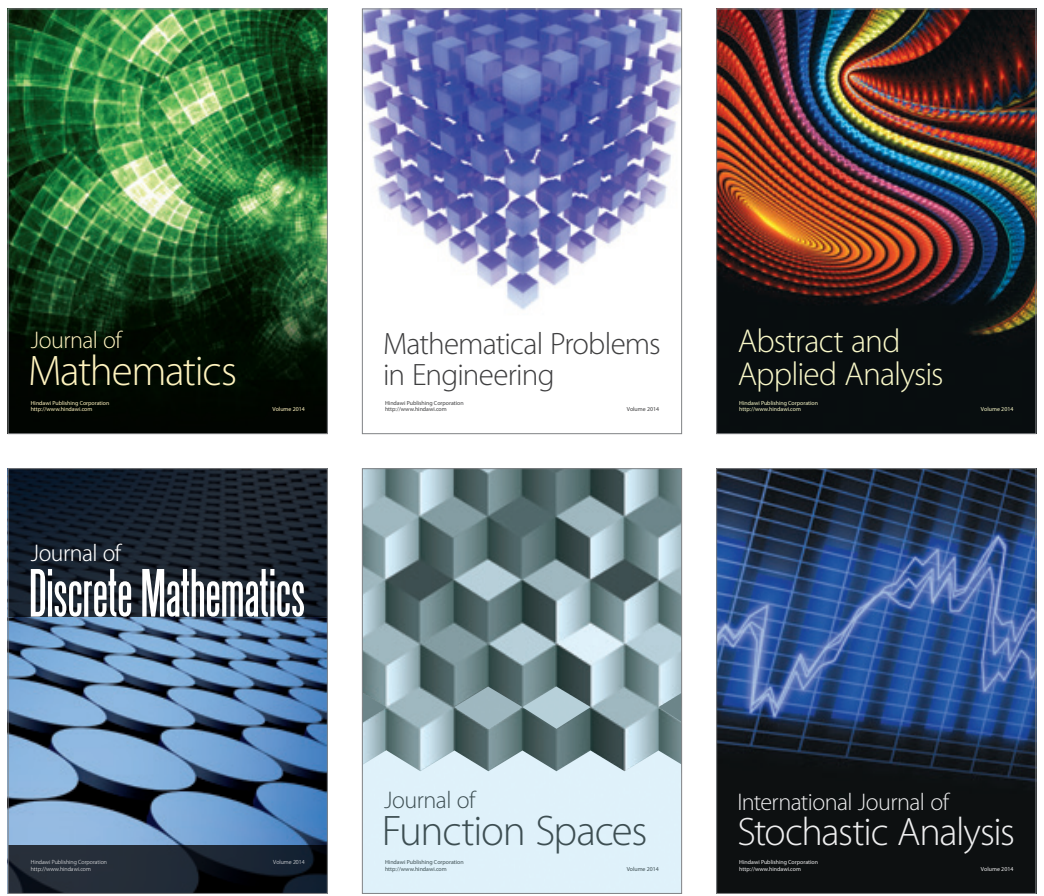

Journal of

Function Spaces

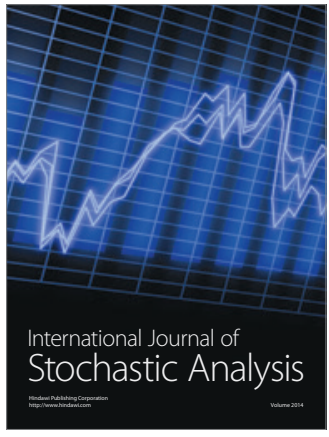

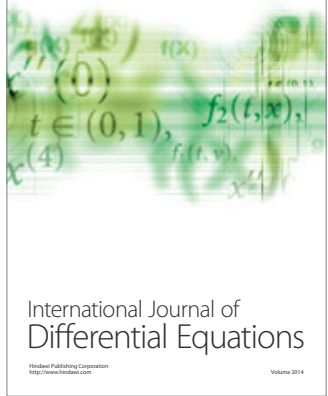
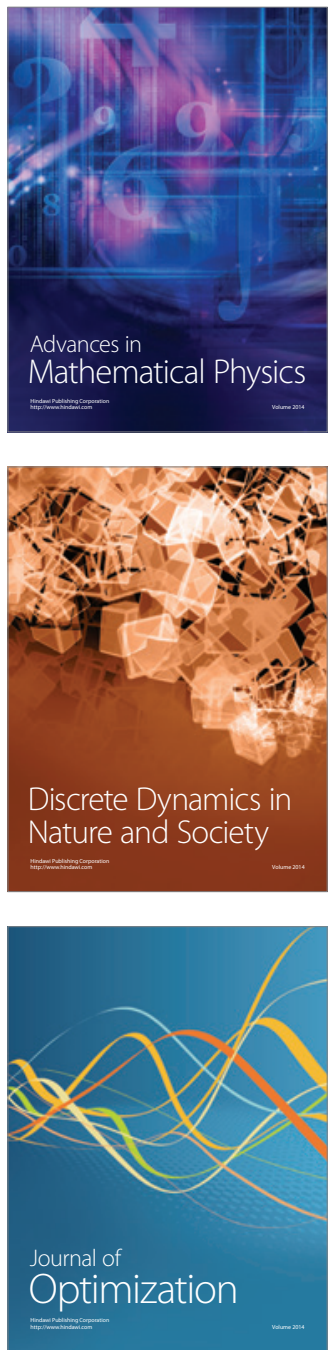